Poly(ethylene oxide) melt intercalation in graphite oxide: sensitivity to topology, cyclic versus linear chains

Fabienne Barroso-Bujans ${ }^{* a, b, ~ c, ~ d ~ J u ̈ r g e n ~ A l l g a i e r, ~}{ }^{\mathrm{e}}$ and Angel Alegria, ${ }^{\mathrm{a}, \mathrm{d}}$

aMaterials Physics Center, CSIC-UPV/EHU, Paseo Manuel Lardizábal 5, San Sebastian 20018, Spain

bDonostia International Physics Center (DIPC), Paseo Manuel Lardizábal 4, San Sebastian 20018, Spain

'IKERBASQUE - Basque Foundation for Science, María Díaz de Haro 3, E-48013 Bilbao, Spain

dDepartamento de Física de Materiales, University of the Basque Country (UPV/EHU), Apartado 1072, San Sebastian 20080, Spain

e Jülich Centre for Neutron Science (JCNS-1) and Institute for Complex Systems (ICS-1), Forschungszentrum Jülich GmbH, 52425 Jülich, Germany

\title{
Methods
}

\section{Synthesis of cyclic PEO}

Linear PEOs containing two hydroxyl end groups were used for generating cyclic PEOs of different molecular weights. The cyclization reactions were carried out with the help of tosyl chloride in the presence of $\mathrm{KOH}$. In this process, intermediately formed tosylated chain ends react with still unreacted $\mathrm{OH}$-end groups under the formation of ether bonds. In a diluted solution and considering that the ring closure reaction is fast compared to the tosylation reaction, predominantly unimeric rings are formed by reaction of a tosylated chain end with the still OH-functionalized second chain end. As the samples were synthesized in gram quantities, the ring closure reactions took place at moderate dilution conditions which lead to chain coupling. Unreacted linear precursor and chain coupled higher molecular weight linear byproducts were eliminated from the cyclization raw products by oxidizing the alcoholic chain ends to carboxylic acids and removing the oxidized material with the help of a basic ion exchange resin. Higher molecular weight cyclic PEO was removed by fractionation using chloroform/heptane as solvent/nonsolvent pair.

The characterization results of linear and cyclic PEO samples are summarized in Table $\mathrm{S} 1$. The SEC traces of cyclic PEOs are shown in Figure S1. A detailed description of the synthetic and characterization procedures is given in reference $\mathrm{S} 1$. 
Table S1. Characterization of cyclic PEOs and their linear precursors. The values for $M_{n}$ and $M_{w} / M_{n}$ were measured by SEC and the contents of linear PEO were quantified by ${ }^{1} \mathrm{H}-\mathrm{NMR}$.

\begin{tabular}{c|cc|cc}
\hline Name & \multicolumn{2}{|c|}{ linear PEO } & \multicolumn{2}{c}{ cyclic PEO } \\
& $\mathbf{M}_{\mathbf{n}}$ & $\mathbf{M}_{\mathbf{w}} / \mathbf{M}_{\mathbf{n}}$ & $\mathbf{M}_{\mathbf{w}} / \mathbf{M}_{\mathbf{n}}$ & residual linear PEO \\
\hline $2 \mathrm{k}$ & 1.860 & 1.03 & 1.02 & $0.3 \%$ \\
$5 \mathrm{k}$ & 5.600 & 1.01 & 1.01 & $0.4 \%$ \\
$10 \mathrm{k}$ & 10.900 & 1.02 & 1.02 & $1.0 \%$ \\
$20 \mathrm{k}$ & 20.300 & 1.01 & 1.03 & $3.7 \%$ \\
\hline
\end{tabular}

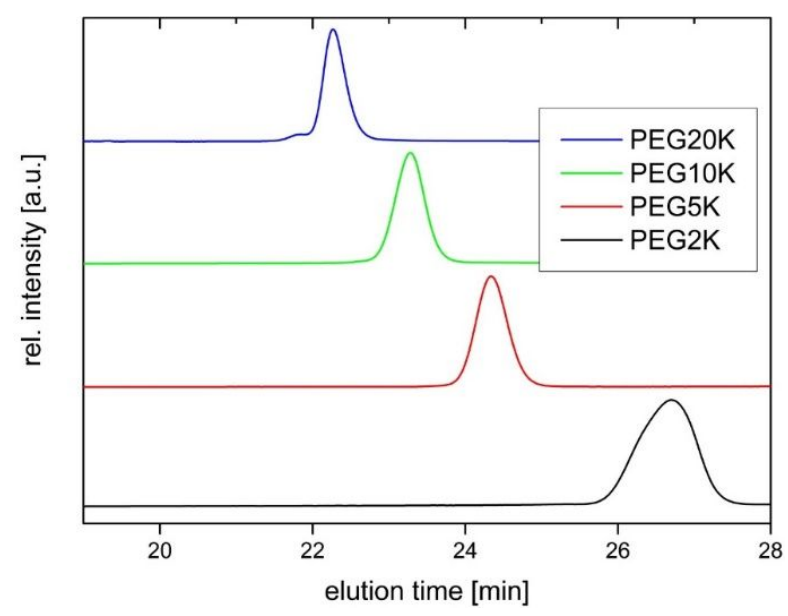

Figure S1. SEC chromatograms of cyclic PEO samples.

\section{Synthesis of $G O$}

A reaction flask containing $200 \mathrm{~mL}$ of fuming nitric acid (Fluka) was cooled to $0{ }^{\circ} \mathrm{C}$ for 20 min using a cryostat bath, followed by the immersion of $10 \mathrm{~g}$ of graphite. Then, $80 \mathrm{~g}$ of potassium chlorate (Fluka) was slowly added over a period of $1 \mathrm{~h}$ in order to avoid sudden increases in temperature. The reaction mixture was stirred for $21 \mathrm{~h}$ at $0{ }^{\circ} \mathrm{C}$. The mixture was then diluted in distilled water, filtered and washed with water until the supernatant had a nitrate content below $1 \mathrm{mg} / \mathrm{L}$ (AQUANAL-plus nitrate). The resulting GO slurry was dried at $80{ }^{\circ} \mathrm{C}$ for $24 \mathrm{~h}$ in a vacuum oven, and stored in vacuum at room temperature until further use.

Figure S2 displays the C1s XPS spectrum of GO and Table S2 shows the binding energy of core levels and $\mathrm{O} / \mathrm{C}$ ratio of $\mathrm{GO}$. The peak was decomposed into a linear combination of two symmetric components, as previously reported in the literature. ${ }^{\mathrm{S} 2}$ The peak at $284.8 \mathrm{eV}$ (relative peak area 45\%) corresponds to $\mathrm{sp}^{2} \mathrm{C}-\mathrm{C}$ bonds in graphitic carbon 
whereas that at $286.5 \mathrm{eV}$ (relative peak area 55\%) corresponds to epoxy and hydroxyl C-O bonds.

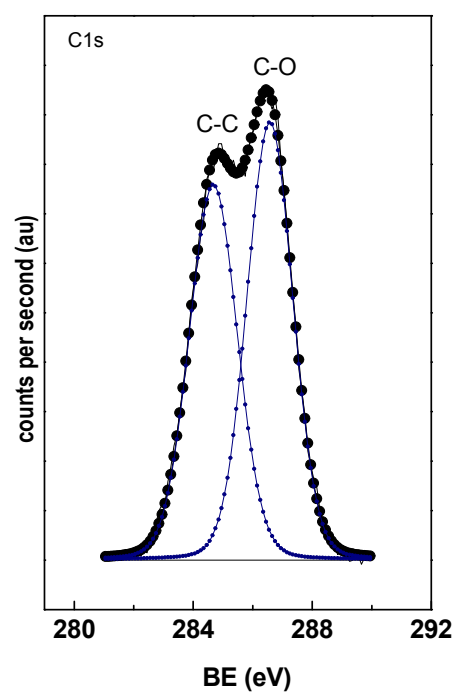

Figure S2. C1s core-level spectra of GO.

Table S2. Binding energy (eV) of core levels and $\mathrm{O} / \mathrm{C}$ ratio of $\mathrm{GO}$.

\begin{tabular}{l|l|l}
\hline C1s & O1s & O/C \\
\hline $284.8(47)$ & & \\
$286.6(53)$ & 532.3 & 0.364 \\
\hline
\end{tabular}

Figure S3 exhibits the solid-state ${ }^{13} \mathrm{C}$ magic-angle spinning NMR spectrum of GO. Three main peaks were clearly detected at about 127, 68 and $54 \mathrm{ppm}$. Following the work of Lerf et al., ${ }^{\mathrm{S} 3}$ these signals were assigned to $\mathrm{C}=\mathrm{C}, \mathrm{C}-\mathrm{OH}$, and epoxide 1,2-ether, respectively. No sign of $\mathrm{C}=\mathrm{O}$ signals were found in the range of 160-200 ppm.

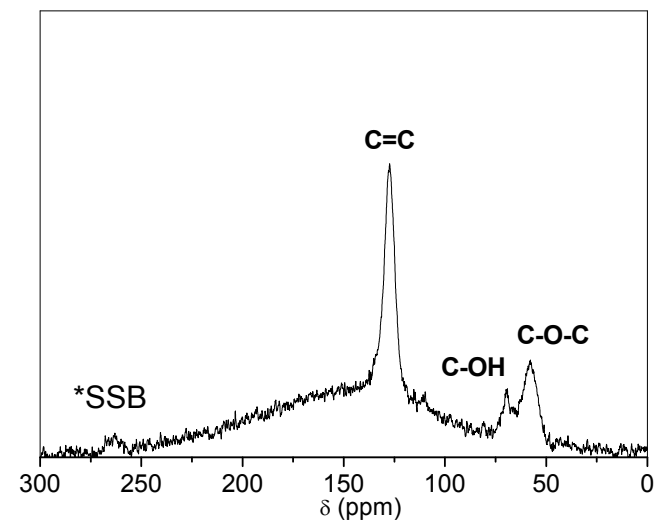

Figure S3. Solid-state ${ }^{13} \mathrm{C}$ magic-angle spinning NMR spectrum of GO. ( $\left.{ }^{*}\right)$ Spinning side bands (SSB). 


\section{Characterization techniques}

The linear PEO precursors and the cyclic products were characterized by SEC using a Polymer Laboratories PL 220 SEC instrument equipped with a differential refractive index detector and with three PolyPore columns at $50{ }^{\circ} \mathrm{C}$. The solvent was a mixture of THF and DMA (85:15 by volume) at a flow rate of $1 \mathrm{~mL} / \mathrm{min}$. For the examination of samples, containing carboxylated PEO, 1 vol- $\%$ of acetic acid was added to the solvent mixture. PEO standards were used for calibration.

The concentration of $\mathrm{OH}$ and $\mathrm{COOH}$ end groups in the cyclic products was determined by NMR using a Bruker Avance III $600 \mathrm{MHz}$ spectrometer equipped with a Prodigy cryoprobe with a $5 \mathrm{~mm}$ PFG AutoX DB probe. The polymer samples were dissolved in pyridine- $\mathrm{d}_{5}$ at concentrations of 10 to $15 \mathrm{mg} / \mathrm{mL}$ and measured at $295 \mathrm{~K}$. In order to quantify the small end group signals, 512 scans were collected at a delay time of $25 \mathrm{~s}$. In none of the samples $\mathrm{COOH}$ groups could be detected but small concentrations of $\mathrm{OH}$ groups indicating smaller quantities of residual linear precursor.

${ }^{13} \mathrm{C}$ magic-angle-spinning (MAS) NMR spectra of GO were recorded using a Bruker Avance DSX300 spectrometer operating at $74.488 \mathrm{MHz}$ and a $7 \mathrm{~mm}$ MAS probe at room temperature (about $22{ }^{\circ} \mathrm{C}$ ). ${ }^{13} \mathrm{C}$ Bloch decay spectra with high-power $(30 \mathrm{kHz}){ }^{1} \mathrm{H}$ decoupling were recorded with spinning at $7.5 \mathrm{kHz}, 90^{\circ}$ pulses of $7.5 \mu$ s duration, and 20 s recycle delays.

Elemental analysis of pristine GO was performed with a Euro EA3000 Elemental Analyzer (CHNS). To check the reproducibility of our procedure, two independent measurements were carried out.

X-ray photoelectron spectroscopy XPS spectra of GO were recorded using an Escalab 200R spectrometer equipped with a hemispherical analyzer and operating in a constantpass energy mode with an un-monochromatized $\mathrm{MgK} \alpha \mathrm{X}$-ray source ( $\mathrm{hv}=1253.6 \mathrm{eV}$ ) powered at $10 \mathrm{~mA}$ and $12 \mathrm{kV}$. The binding energies (BE) were referenced to the $\mathrm{C} 1 \mathrm{~s}$ peak at $284.9 \mathrm{eV}$. Data processing was performed with the XPS Peak program. The spectra were decomposed with the least-squares fitting routine provided by this software using a Gauss/Lorentz product function and a Shirley background. 


\section{Supplementary figures}
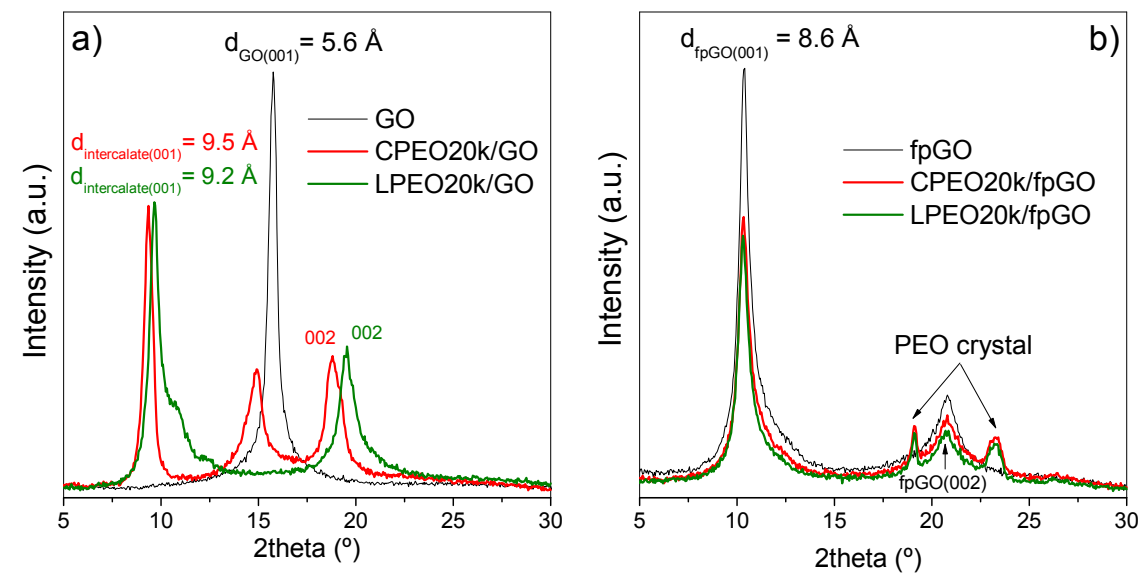

Figure S4. XRD profiles registered at $25^{\circ} \mathrm{C}$ of (a) pristine $\mathrm{GO}$ and intercalated hybrid samples, CPEO20k/GO and LPEO20k/GO, recovered at the end of the experiment from the DSC pan used for in situ intercalation; and (b) fully-pillared GO (fpGO) and samples (CPEO20k/fpGO and LPEO20k/fpGO) recovered at the end of the experiment from the DSC pan used for in situ intercalation.
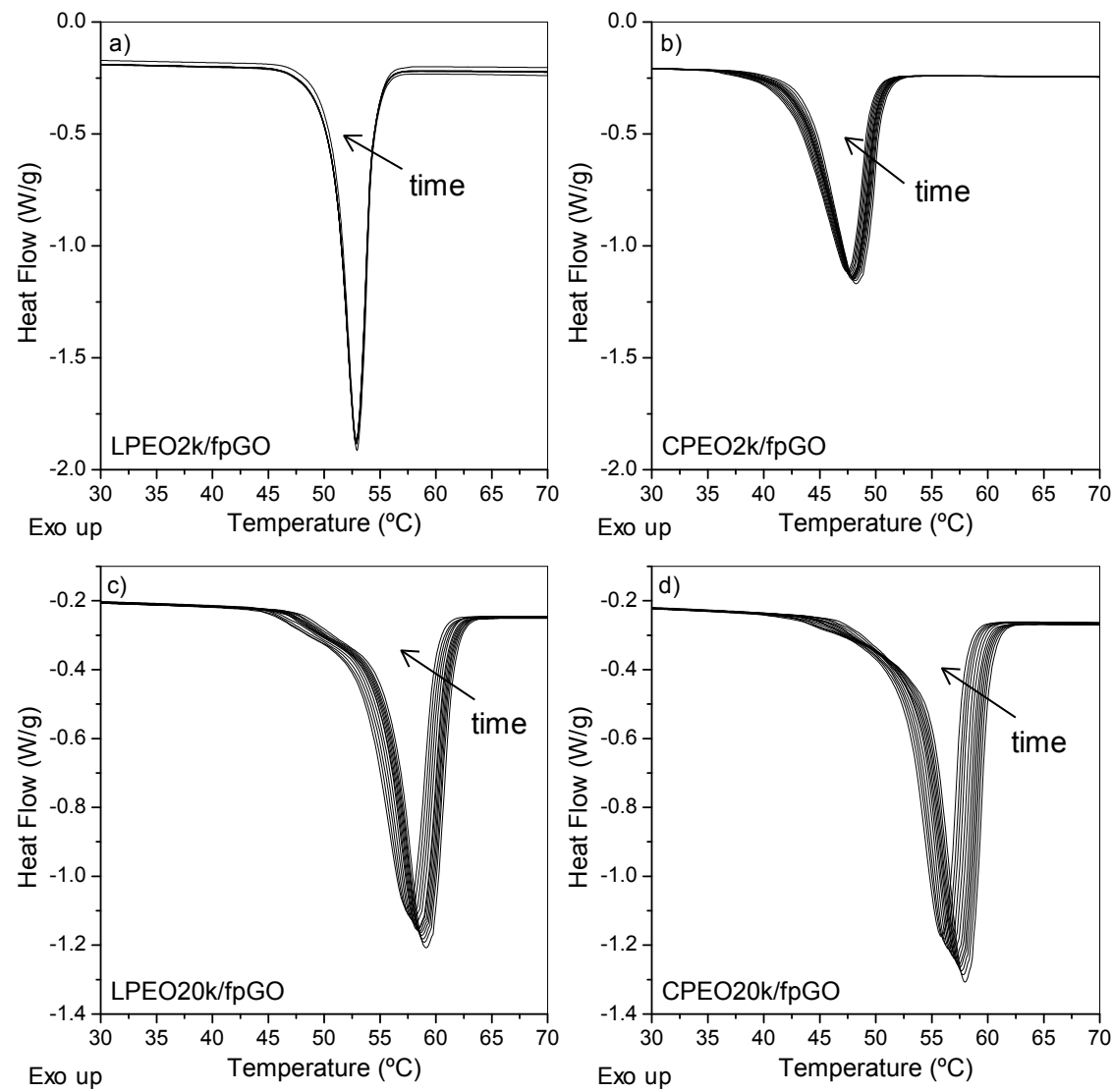

Figure S5. DSC thermograms of annealed samples at $80{ }^{\circ} \mathrm{C}$ by using a fpGO/PEO ratio of $82 / 18 \mathrm{wt} \%$. Heating and cooling were recorded at a rate of $10{ }^{\circ} \mathrm{C} / \mathrm{min}$. 

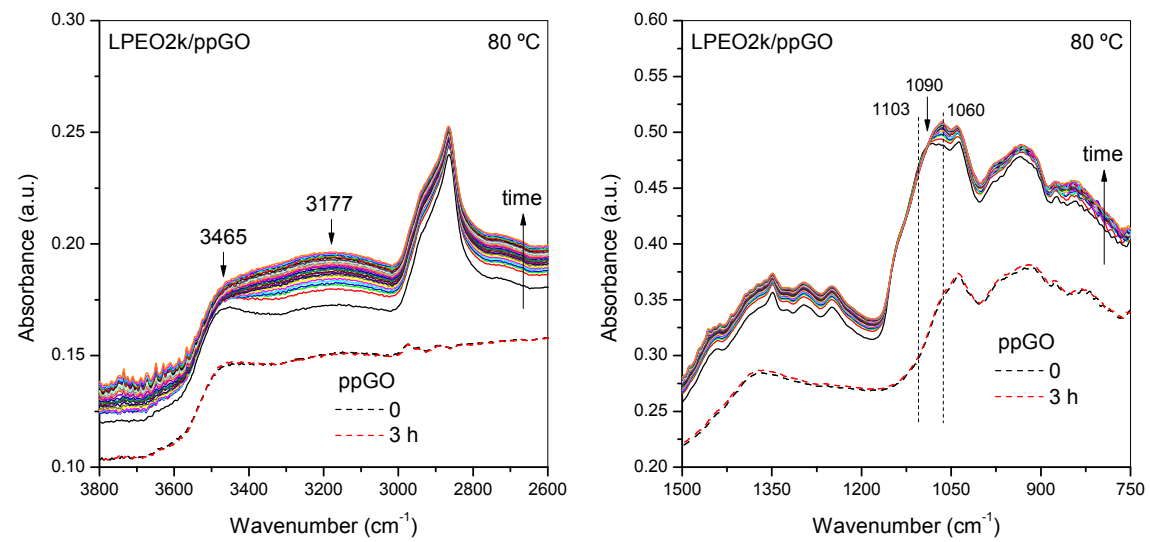

Figure S6. ATR-FTIR data of LPEO2k/ppGO and ppGO annealed in situ at $80{ }^{\circ} \mathrm{C}$ for 3 $\mathrm{h}$. To simplify, only the data recorded at the beginning and after $3 \mathrm{~h}$ are represented for ppGO.
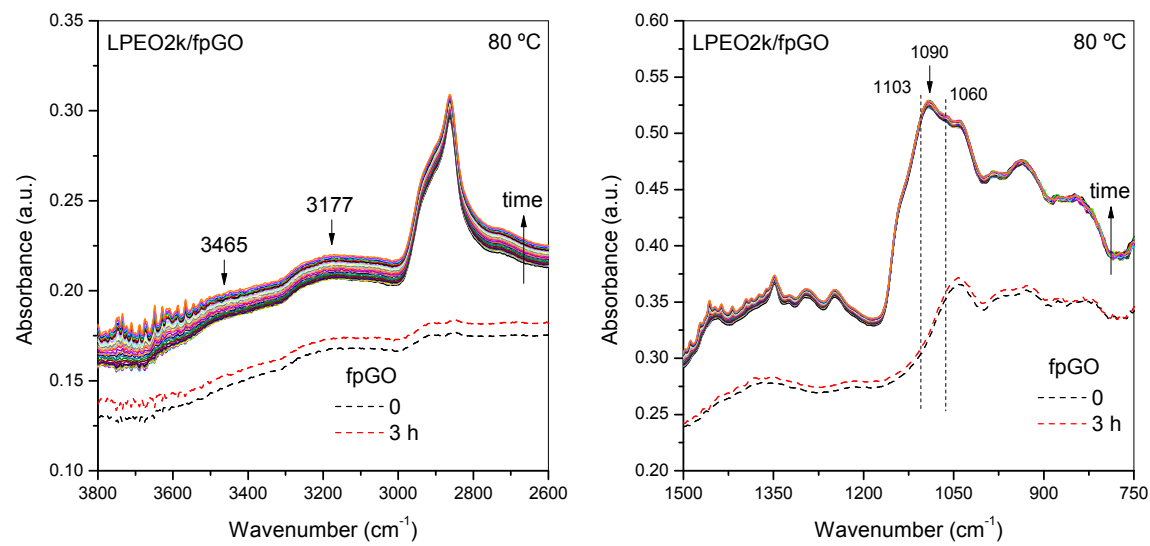

Figure S7. ATR-FTIR data of LPEO2k/fpGO and fpGO annealed in situ at $80{ }^{\circ} \mathrm{C}$ for $3 \mathrm{~h}$. To simplify, only the data recorded at the beginning and after $3 \mathrm{~h}$ are represented for fpGO.
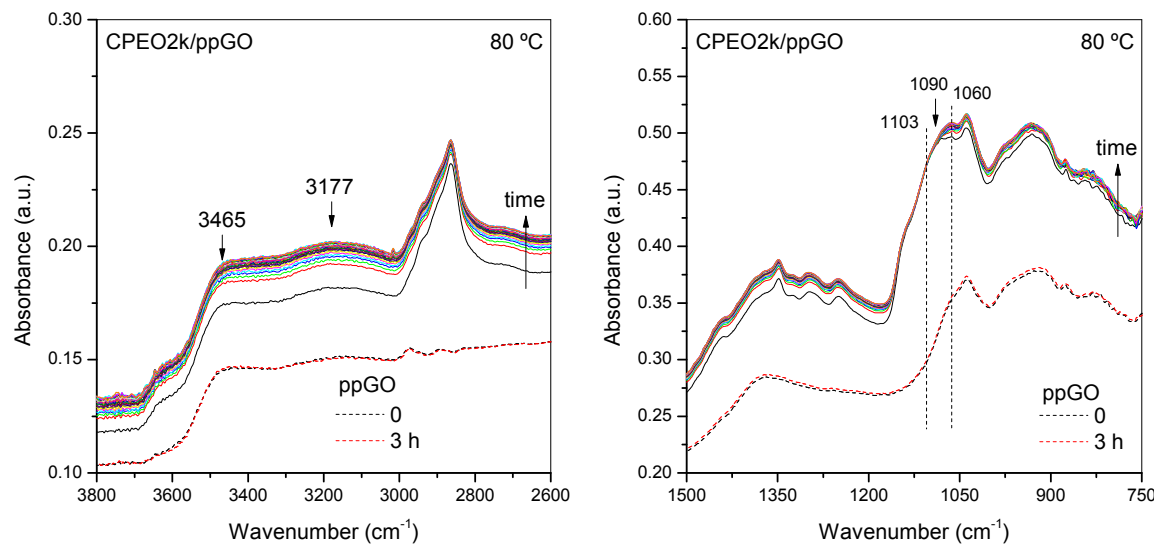

Figure S8. ATR-FTIR data of CPEO2k/ppGO and ppGO annealed in situ at $80{ }^{\circ} \mathrm{C}$ for 3 $\mathrm{h}$. To simplify, only the data recorded at the beginning and after $3 \mathrm{~h}$ are represented for ppGO. 


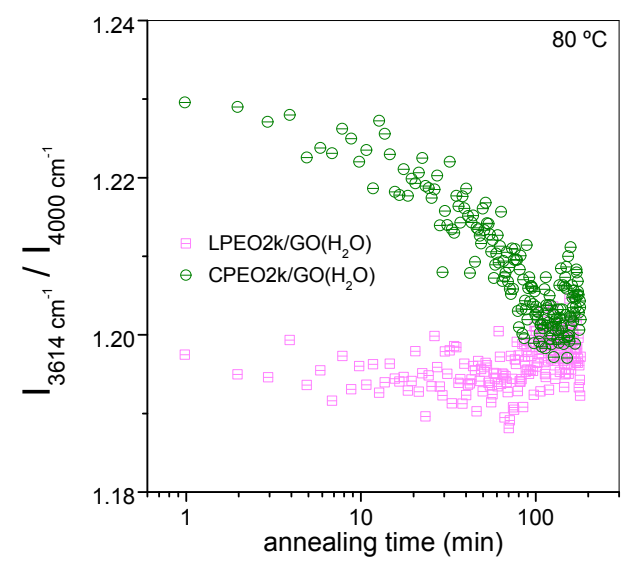

Figure S9. Evolution of the relative peak intensity at $3614 \mathrm{~cm}^{-1}$ assigned to weakly hydrogen-bonded water as a function of annealing time.

\section{References}

(S1) Hövelmann, C. H.; Gooßen, S.; Allgaier, J., Scale-Up Procedure for the Efficient Synthesis of Highly Pure Cyclic Poly(ethylene glycol). Macromolecules 2017, 50, (11), 4169-4179.

(S2) Yang, D.; Velamakanni, A.; Bozoklu, G.; Park, S.; Stoller, M.; Piner, R. D.; Stankovich, S.; Jung, I.; Field, D. A.; Ventrice Jr, C. A.; Ruoff, R. S., Chemical analysis of graphene oxide films after heat and chemical treatments by X-ray photoelectron and Micro-Raman spectroscopy. Carbon 2009, 47, (1), 145-152.

(S3) Lerf, A.; He, H.; Forster, M.; Klinowski, J., Structure of Graphite Oxide Revisited. J. Phys. Chem. B 1998, 102, (23), 4477-4482. 